\title{
Patrones de abundancia y riqueza de componentes de la costra biológica del suelo en un matorral seco del sur de Ecuador
}

\author{
Andrea P. Castillo-Monroy ${ }^{1 *}$, Ángel Benítez ${ }^{2}$ \\ ${ }^{1}$ Universidad Técnica Particular de Loja, Departamento de Ciencias Naturales, San Cayetano Alto, Loja, Ecuador \\ ${ }^{2}$ Universidad Técnica Particular de Loja, Museo de Colecciones Biológicas (MUTPL), Loja, Ecuador \\ *Autor para correspondencia, correo electrónico: apcastillo4@utpl.edu.ec \\ Editado por/Edited by: Diego F. Cisneros-Heredia, PhD.(c) \\ Recibido/Received: 2015/01/27. Aceptado/Accepted: 2015/05/11 \\ Publicado en línea/Published online: 2015/05/22. Impreso/Printed: 2015/06/01

\section{Abundance patterns and components richness of biological soil crust in a dry shrubland in southern Ecuador}

\begin{abstract}
Biological soil crusts (BSCs) result from an intimate association between soil particles and cyanobacteria, algae, microfungi, lichens, and bryophytes. Important advances in the understanding of BSC and their key role in ecosystem processes in dry lands are widely known. However, little is known about the small-scale patterns of abundance and distribution of BSC-forming lichens, mosses and cyanobacteria in South America, particularly in Ecuador. In order to fill this important information gap, we set to identify patterns of abundance and richness of mosses and lichens forming BSC along the elevation gradient of dry southern Ecuador. To reach our objective, we identify three levels at 1400,1500 and $1600 \mathrm{~m}$ elevation. At each level, we selected 180 subplots (60 per elevation level). Each subplot $(25 \times 25 \mathrm{~cm}$ quadrat) was divided into $5 \times 5 \mathrm{~cm}$, spread over a homogeneous area of 1.5 ha. The richness and cover of species forming BSC (lichens, mosses and cyanobacteria) were estimated. We conducted a variance analysis to evaluate the effect of elevation on the richness and diversity of species. We found 24 species in the three elevation levels, sixteen lichens (two are first reports for Ecuador), five mosses, two cyanobacteria, and one pteridophyta. Our results clearly show that the species richness of BSC respond to elevation. Our research becomes the first report of BSC in Ecuador and adds new information on the abundance and species richness of BSC.
\end{abstract}

Keywords. Cyanobacteria, diversity, ecology, elevation gradient, lichens, mosses, species richness.

\section{Resumen}

Importantes avances en el conocimiento del rol ecológico de la costra biológica del suelo (i.e. CBS; comunidad biótica formada por la íntima asociación entre partículas de suelo, cianobacterias, algas, hongos, líquenes, hepáticas y musgos) y su papel fundamental en los procesos de ecosistemas secos del mundo, son ampliamente conocidos. Sin embargo, poco se sabe acerca de los patrones de abundancia y distribución de los líquenes y musgos que forman la CBS en Sur América, particularmente en Ecuador. Con el fin de llenar este importante vacío de información, nos planteamos describir la diversidad de líquenes, musgos y cianobacterias que conforman la CBS a lo largo de un gradiente altitudinal en un matorral seco del sur de Ecuador. Para alcanzar nuestro objetivo seleccionamos tres niveles de elevación a lo largo de un gradiente altitudinal (i.e. 1400, 1500 and $1600 \mathrm{~m}$ ). Muestreamos un total de $180 \mathrm{sub}$-parcelas $(25 \times 25 \mathrm{~cm})$. Cada sub-parcela fue dividida en 25 cuadrantes $(5 \times 5 \mathrm{~cm})$, donde se registró la presencia y cobertura de todas las especies (líquenes, musgos, cianobacterias). Realizamos un análisis de varianza para evaluar el efecto de la elevación sobre la riqueza y abundancia de especies. Encontramos 24 especies en lo largo del gradiente; 16 líquenes (de las cuales dos son primer reporte para Ecuador), 5 musgos, 2 cianobacterias y 1 planta vascular. Nuestros resultados indican que el número total de especies aumenta con el incremento de la elevación. Nosotros reportamos por primera vez la presencia de la CBS en Ecuador y aportamos nueva información sobre el conocimiento de la diversidad y riqueza de especies de la CBS.

Palabras clave. Cianobacterias, diversidad, ecología, gradiente altitudinal, líquenes, musgos, riqueza de especies. 


\section{Introducción}

La costra biológica del suelo (CBS) es una comunidad conformada por briofitos (musgos y hepáticas), líquenes, bacterias, cianobacterias, hongos microscópicos y algas verdes [1-2], en una íntima asociación con las partículas de suelo en los primeros milímetros de la superficie. La CBS cumple varias funciones ecológicas, principalmente en ecosistemas secos, favoreciendo la fijación de nitrógeno y carbono [3-4], ayudando contra la acción erosiva de la lluvia y el viento [5], y modulando la mineralización de nutrientes, el carbono liberado por la respiración del suelo, el balance de la escorrentía, y la infiltración del agua [6-9]. La CBS también influye en la distribución y abundancia de plantas, animales y microorganismos del suelo [10-12]. El papel funcional y la distribución de la CBS ha sido bien documentada en la última década. Sin embargo, la mayoría de las investigaciones se centran en las regiones áridas y semiáridas de las zonas subtropicales (EE.UU. [1], España [13], Australia [8]), con algunas investigaciones desarrolladas en regiones templadas [14] y muy pocas documentadas en regiones tropicales [15-16]. Las zonas secas de América del Sur presentan condiciones climáticas y edáficas óptimas para el desarrollo de la CBS [1,17], de ahí que puede ser un componente biótico de primera magnitud en los suelos de dichos ecosistemas [17]. Las investigaciones sobre la CBS en el trópico americano son escasas, no obstante existe registro de la presencia de la CBS en Venezuela [18] y Colombia (A. P. Castillo-Monroy, obs. pers.). Los cambios altitudinales en la diversidad de algunos componentes de la CBS han sido estudiados de manera independiente (ej. musgos, [22]; líquenes, [23] y cianobacterias, [24]), pero no se conoce trabajos que los hayan estudiado en conjunto como una comunidad. De ahí que nos planteáramos describir la diversidad de líquenes, briofitos y cianobacterias a lo largo de un gradiente altitudinal de un matorral seco. Nuestros resultados establecerán la línea base sobre la biodiversidad macroscópica que conforma la CBS, lo cual permitirá una mejor comprensión, en subsiguientes investigaciones, de su ecología en Ecuador.

\section{Materiales y métodos}

\section{Área de estudio}

El estudio se llevó a cabo en un matorral seco localizado en la finca Alamana, cantón Catamayo, provincia de Loja, sureste de Ecuador (674634 E, 9557997 N; Fig. 1). El clima es seco tropical con una pronunciada estación seca que va de mayo a diciembre; temperatura, evapotranspiración y precipitación media anual es de



Figura 1. Área de estudio finca Alamala, Cantón Catamayo, Provincia de Loja, sureste de Ecuador. Los símbolos (cuadrado, circulo, triangulo) indican las parcelas en los tres niveles de elevación. 
$27,5^{\circ} \mathrm{C}, 1112 \mathrm{~mm}$ y $382 \mathrm{~mm}$, respectivamente [26]. La vegetación está dominada por un complejo de arbustos de hoja perene (Croton spp. aff. thurifer Kunth y Croton sp. aff. ferrugineus Kunth) de hasta $2 \mathrm{~m}$ de altura, cubriendo el $40 \%$ de la cobertura total y formando parches intercalados sobre toda el área. Otras especies comunes son: Euphorbia weberbaueri Mansf., Repert. Spec [27] y Acacia macracantha Humb. \& Bonpl. ex Willd., [28]. La CBS y una planta pteridofita perenne, Selaginella sellowii Hieron, de habito rastrero, están presente en las áreas desprovistas de vegetación arbustiva (Fig. 2) y representan $15-18 \%$ de la cobertura total.

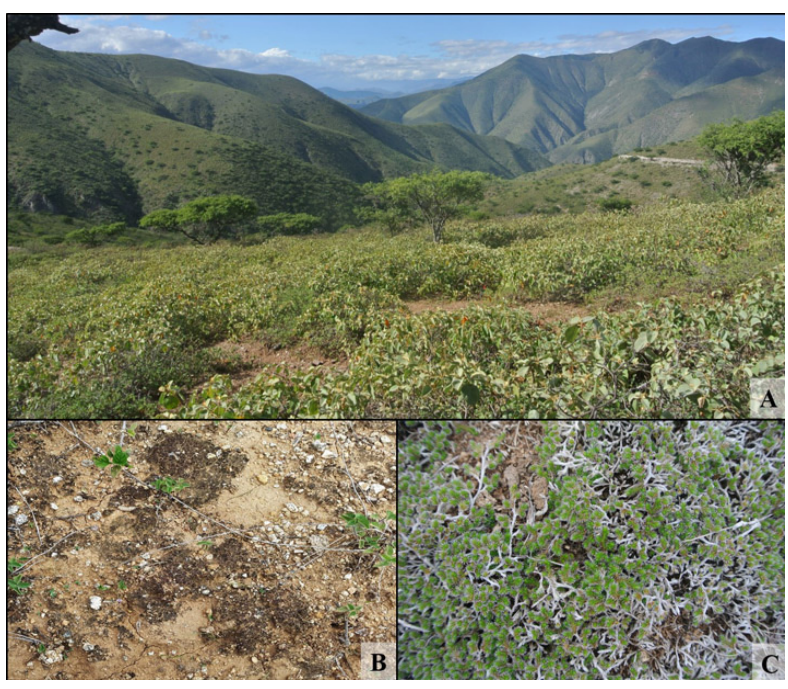

Figura 2. Vista panorámica de matorral seco de la finca Alamana, Cantón Catamayo (A). Las áreas entre parches de vegetación corresponden a la costra biológica del suelo dominada principalmente por líquenes (B) y Selaginella sellowii $(\mathbf{C})$

\section{Diseño y recolección de datos}

Se seleccionó tres niveles de elevación (nivel 1: 1600 m; nivel 2: 1500 m, y nivel 3: 1400 m). En cada nivel se estableció tres parcelas de $10 \times 10 \mathrm{~m}$ con similares características de vegetación perenne (plantas espermatofitas arriba mencionadas), orientación (OE) y pendiente $(>5 \%)$. En cada parcela se estableció 20 sub-parcelas de $25 \times 25 \mathrm{~cm}$. Cada sub-parcela fue dividida en cuadrantes de $5 \times 5 \mathrm{~cm}$. Las sub-parcelas fueron distribuidas noaleatoriamente en las parcelas con el fin de garantizar la presencia de CBS. La distancia mínima de separación entre sub-parcelas fue de $1,5 \mathrm{~m}$ para reducir el riesgo de dependencia entre las sub-parcelas [29, 30]. En total se colocó 180 sub-parcelas donde se estimó la riqueza, frecuencia, cobertura y diversidad de cada uno de los organismos que la conforman (briofitos, líquenes y cianobacterias). La cobertura total se estimó como el promedio de cada especie presente en la sub-parcela, la riqueza como el número de especies presentes en cada cuadrante de las sub-parcelas y la diversidad de especies fue estimada usando el Índice de Shannon-Wiener (H'). La cobertura se utilizó como un sustituto de la abundancia debido a las dificultades inherentes a la definición de especie y porque es un buen estimador de la biomasa de los organismos que conforman la CBS [31]. Todas las especies de líquenes, briofitos y cianobacterias que aparecieron en las sub-parcelas fueron colectadas, procesadas e identificadas en Herbario de Colecciones Biológicas de la Universidad Técnica Particular de Loja (HUTPL). Para la colección e identificación de briofitos se utilizó los protocolos o estándares internacionales señalados en la Guía de Briofitos de América Tropical [32]. La nomenclatura para briofitos sigue a Léon-Yánez et al. [33] en el caso de las especies de hepáticas y Churchill et al. [34] para los musgos. Para líquenes y cianobacterias se utilizó los protocolos señalados por Nash et al. [35]. Se examinó caracteres macroscópicos, tales como el color y estructura del talo, así como estructuras reproductoras como apotecios y peritecios bajo un estereoscopio de hasta 50× magnificación. Para los caracteres anatómicos como estructura del talo, tipo de esporas y otras estructuras se hizo cortes con hoja de afeitar y se examinó bajo un microscopio con aumentos de $10 \times, 40 \times$ y $100 \times$. Además se utilizó sustancias químicas como $\mathrm{K}$ (solución de hidróxido de potasio al 10\%) y C (lejía comercial) bajo el estereoscopio para observar las reacciones de color de talo y estructuras reproductoras (ascomas). La nomenclatura para las especies sigue al MycoBank (http:// www.mycobank.org/).

\section{Análisis de datos}

La riqueza total en cada nivel de elevación se comparó mediante el estimador de riqueza no paramétrico Chao2, con el programa EstimateS 9.1.0 [36]. Para analizar los cambios en la riqueza y diversidad (H') de especies en cada nivel de elevación se utilizó un análisis de varianza de una vía (ANOVA). Previo a la ANOVA, se confirmó la asunción de homogeneidad y normalidad de las varianzas. Los datos se log-transformaron cuando fue necesario. Todos los análisis fueron realizados con el programa estadístico SPSS 16.0 (SPSS Inc., Chicago, IL, USA).

\section{Resultados}

Se identificó un total de 24 especies, incluyendo 16 líquenes, 5 musgos y 2 cianobacterias, distribuidos en 17 géneros y 13 familias (Tabla 1). Dos especies de líquenes, Peltula patellata (espécimen HUTPLAB-648) y 
Placidium pilosellum (HUTPLAB-649), se reportan por primera vez para Ecuador (Fig. 3). Un total de 20 especies fueron identificadas a $1600 \mathrm{~m}$, y 18 y 15 especies a 1500 y 1400, respectivamente. Un patrón similar fue observado por el estimador de riqueza Chao 2 confirmando un alto número de especies a $1600 \mathrm{~m}$ al compararlo con los demás niveles (Tabla 2). Selaginella sellowii fue más abundante que todas las especies de musgo, pero menos abundante que las dos cianobacterias y que la sumatoria de las abundancias de todas las especies de líquenes en los tres niveles de elevación. Las forma de crecimiento predominante de los líquenes fue escuamuloso, seguido de folioso / gelatinoso y finalmente el crustáceo. Las familias Verrucariaceae y Pottiaceae presentaron el mayor número de especies (4 especies), seguida de Collemantaceae y Peltulacea con 3 especies cada una, las demás familias presentan un menor número de especies. Los géneros más representativos fueron Collema, Peltula y Placidimun (2-3 especies). Encontramos diferencias significativas cuando analizamos la riqueza y diversidad $\left(\mathrm{H}^{\prime}\right)$ de especies de la CBS $\left(\mathrm{F}_{2-177}=9486,05, \mathrm{P}=<\right.$ $0,001, \mathrm{~F}_{2-177}=5225,75, \mathrm{P}=<0,001$; respectivamente). La riqueza aumentó a media que incrementó la elevación, con diferencias significativas entre los tres niveles de elevación. La diversidad ( $\left.\mathrm{H}^{\prime}\right)$ fue significativamente diferente a $1600 \mathrm{~m}$ con respecto de los otros dos niveles de elevación (Fig. 4).

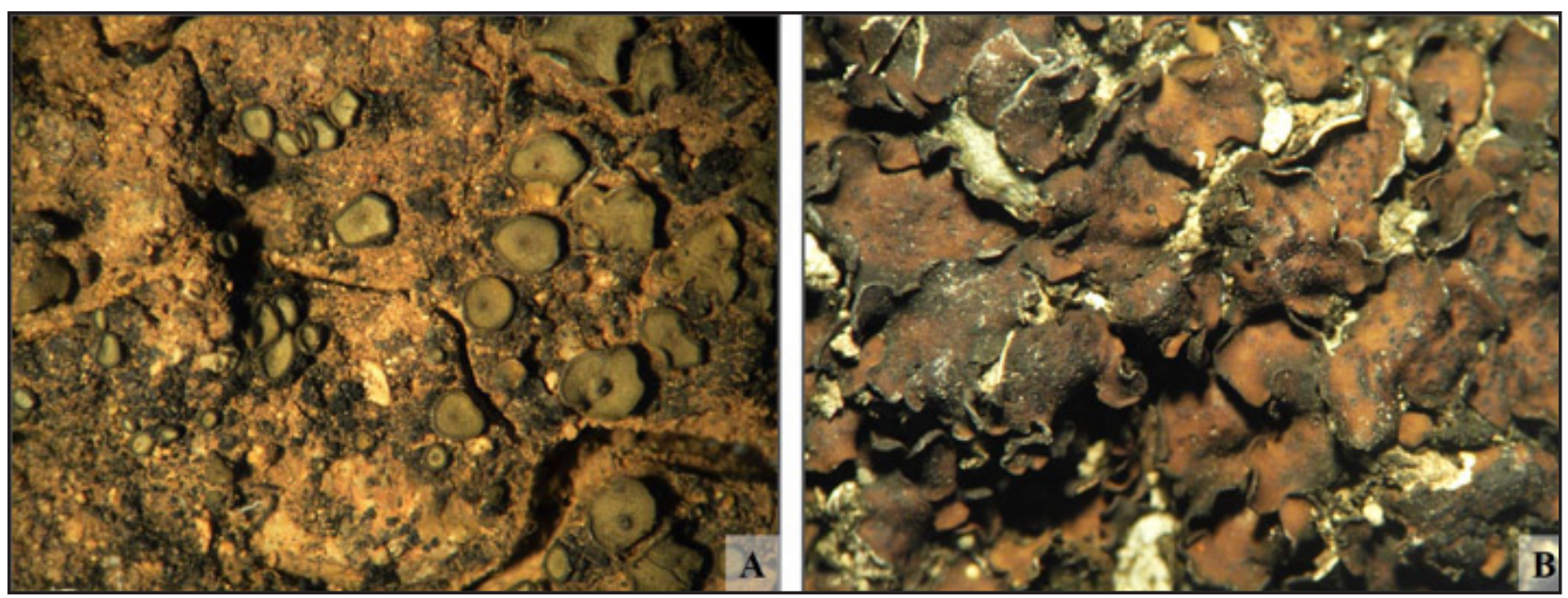

Figura 3. Dos nuevos líquenes registrados para Ecuador; Peltula patellata (A), Placidium pilosellum (B)
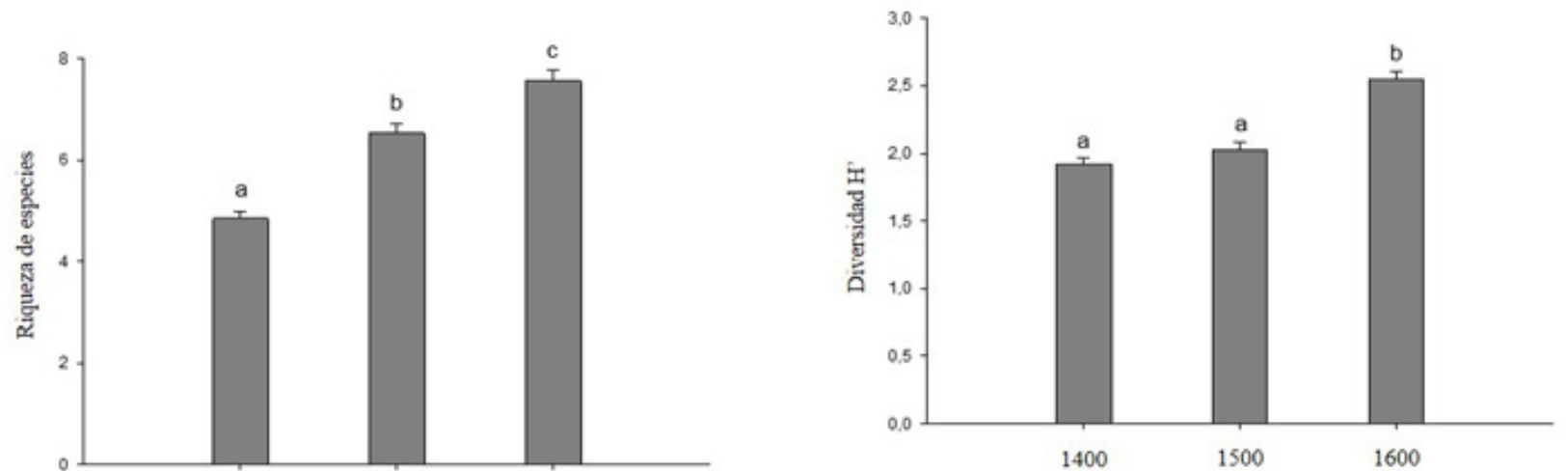

Figura 4. Riqueza y diversidad (H') de especies de la CBS en cada nivel de elevación. Los datos representan medias \pm SD $(n=60)$. 
Tabla 1. Frecuencia (número de veces que ha aparecido una especies en proporción a todos los cuadrantes de la sub-parcela) de las especies que conforman la costra biológica del suelo en los diferentes niveles de elevación.

\begin{tabular}{|c|c|c|c|c|}
\hline \multirow[t]{2}{*}{ Familias } & \multirow[t]{2}{*}{ Especies } & \multicolumn{3}{|c|}{ Frecuencia (\%) } \\
\hline & & $1400 \mathrm{~m}$ & $1500 \mathrm{~m}$ & $1600 \mathrm{~m}$ \\
\hline Pottiaceae & Aloina rigida (Hedw.) Limpr. & 0 & 2 & 0 \\
\hline Bryaceae & Bryum argenteum $\mathrm{Hedw}$ & 20 & 60 & 90 \\
\hline Pottiaceae & Didymodon rigidulus Hedw. & 0 & 3 & 2 \\
\hline Pottiaceae & Tortula fragilis Taylor & 0 & 8 & 15 \\
\hline \multirow[t]{2}{*}{ Pottiaceae } & Weissia controversa Hedw. & 0 & 72 & 43 \\
\hline & Cianobacterias & & & \\
\hline Nostocaceae & $\begin{array}{l}\text { Nostoc commune Vaucher ex Bornet \& } \\
\text { Flahault }\end{array}$ & 67 & 50 & 83 \\
\hline \multirow[t]{2}{*}{ Scytonemataceae } & Scytonema sp. & 88 & 75 & 90 \\
\hline & Líquenes & & & \\
\hline Physciaceae & Buellia sp. aff. punctata (Hoffm.) Mass & 13 & 0 & 0 \\
\hline Collemataceae & Collema sp. 1 & 20 & 0 & 13 \\
\hline Collemataceae & Collema sp. 2 & 0 & 0 & 3 \\
\hline Collemataceae & Collema tenax (Sw.) Ach. & 12 & 78 & 47 \\
\hline Verrucariaceae & Endocarpon pusillum Hedwig & 38 & 78 & 63 \\
\hline Verrucariaceae & Endocarpon sp. & 17 & 0 & 0 \\
\hline Heppiaceae & Heppia despreauxii (Mont.) Tuck. & 29 & 70 & 75 \\
\hline Lecanoraceae & Lecidella sp. & 0 & 0 & 5 \\
\hline Peltulaceae & Peltula obscurans (Nyl.) Gyeln. & 75 & 55 & 57 \\
\hline Peltulaceae & Peltula patellata (Bagl.) Swinscow \& Krog & 0 & 3 & 0 \\
\hline Peltulaceae & Peltula sp. 1 & 5 & 2 & 0 \\
\hline Peltulaceae & Peltula sp. 2 & 0 & 0 & 5 \\
\hline Verrucariaceae & Placidium pilosellum (Breuss) Breuss & 5 & 70 & 67 \\
\hline Verrucariaceae & $\begin{array}{l}\text { Placidium sp. aff. squamulosum (Ach.) } \\
\text { BreuÂ }\end{array}$ & 0 & 13 & 73 \\
\hline Psoraceae & $\begin{array}{l}\text { Psora sp. aff. nipponica (Zahlbr.) Gotth. } \\
\text { Schneider }\end{array}$ & 0 & 17 & 0 \\
\hline \multirow[t]{2}{*}{ Catillariaceae } & Toninia sp. & 5 & 0 & 0 \\
\hline & Pteridofitas & & & \\
\hline Lycopodiaceae & Selaginella sellowii Hieron & 90 & 15 & 5 \\
\hline
\end{tabular}

Tabla 2. Estimador de riqueza de especies (Chao2) que conforman la costra biológica del suelo en los diferentes niveles de elevación.

\begin{tabular}{ccc}
\hline Nivel de elevación & Número de especies observadas & $\begin{array}{c}\text { Número de especies estimadas } \\
\text { Chao } 2\end{array}$ \\
\hline Nivel $\mathbf{3}(\mathbf{1 6 0 0} \mathbf{~})$ & 20 & $22 \pm 0,13$ \\
\hline Nivel $\mathbf{2}(\mathbf{1 5 0 0} \mathbf{~ m})$ & 18 & $20 \pm 1,87$ \\
\hline Nivel $\mathbf{1}(\mathbf{1 4 0 0} \mathbf{~ m})$ & 15 & $17 \pm 0,02$ \\
\hline
\end{tabular}




\section{Discusión}

Nuestro estudio reporta organismos que conforman la CBS por primera vez en Ecuador. Dos nuevas especies de líquenes para Ecuador forman parte de la alta riqueza de especies registrada en nuestra área de estudio (Fig. 3). En total fueron 24 especies, riqueza superada solamente en CBS de España con 41 especies [38] y de México con 34 especies [15]. Sin embargo este último solo reporta ocho especies de líquenes, un número inferior al encontrado en nuestro estudio. Baja diversidad y un menor número de especies ha sido reportado en numerosos estudios; por ejemplo, en Estados Unidos se encontró 13 especies de líquenes, 4 de musgos y una de cianobacterias [39]. En un ecosistema semiárido de España (Aranjuez) 15 especies de líquenes y 5 de musgos fueron reportadas [6], y en el desierto de Monte en Argentina se encontraron 15 especies de líquenes, 5 cianobacterias y un musgo [40]. Cabe resaltar que los estudios antes mencionados han sido desarrollados en zonas templadas que correspondes a zonas más frías que los climas tropicales donde fue desarrollado el presente estudio. Así, nosotros concluimos que las condiciones climáticas propias de los trópicos estaría beneficiando la diversidad de especies de la CBS (hipótesis 1). Esta afirmación será evaluada en futuras investigaciones ya que con el presente diseño experimental y variables estudiadas no es posible llegar a validar dicha hipótesis. Por tanto, hay que tomar este trabajo de manera exploraría y con cautela para evitar generalizaciones erróneas.

Selaginella sellowii fue la especie más abundante a $1400 \mathrm{~m}$, mientras que los musgos lo fueron a $1600 \mathrm{~m}$. Selaginella sellowii, con apariencia y crecimiento de musgo, es una planta vascular tolerante a la desecación debido a la presencia de un azúcar (Trehalosa) que es el responsable de la resistencia a la sequía [41]. Al igual que los musgos, S. sellowii comparte una naturaleza poiquilohídrica, donde en ausencia de agua las actividades fotosintéticas cesan y las plantas pasan a un estado de dormancia [42]. Por lo tanto, aunque S. sellowii y los musgos difieren en su historia evolutiva, nosotros planteamos que ambos grupos de plantas podrían realizar funciones similares, con S. sellowii pudiendo desarrollar los roles ecológicos (captura y almacenamiento de agua en tiempo de máxima sequía, protección del suelos) que desarrollan los musgos (hipótesis 2). Nuestra afirmación se basa en la observación de que $S$ sellowii es más abundante cuando los musgos tienen una menor abundancia, a lo largo del gradiente altitudinal.
En varios trabajos se ha reportado a las cianobacterias como un componente dominante de la CBS. Por ejemplo, Budel et al. [16] reportaron 49 especies en el Karoo suculento y 24 especies en el desierto de Namibia. Ullmann \& Budel [43] reportaron 18 especies en el suroeste de Australia y 13 especies de la fueron reportados por García-Pichel et al. [44] en la meseta de Colorado en América del Norte. El patrón observado en nuestra área de estudio fue diferente al reportado en los trabajos antes mencionados. Nosotros registramos únicamente dos especies, no obstante, son el elemento más común y abundante de la CBS en los tres niveles de elevación. Estas diferencias en la diversidad de las cianobacterias pueden ser debidas a un problema metodológico pues nosotros utilizamos una aproximación morfológica y anatómica para la determinación de especie, la cual presenta una resolución menor en comparación con métodos moleculares utilizados en los estudios antes mencionados.

Los resultados del análisis de varianza muestran claramente que la riqueza de especies responde a un gradiente altitudinal (Fig. 4). Este patrón fue reforzado con los resultados Chao2, los cuales también señalaron una mayor riqueza a elevaciones altas (1600). Nuestros resultados aportan información valiosa sobre la compleja teoría de estructura de comunidades y elevación, donde dependiendo de la latitud, clima, topografía, vegetación, etc., la distribución de las especies a lo largo de un gradiente altitudinal puede incrementar, disminuir, tener forma de campana o no tener tendencia alguna. Varios trabajos han documentado que la riqueza de líquenes y briófitos (especies criptógamas) incrementa a lo largo de un gradiente altitudinal $[45,46]$, similar a nuestros resultados. Sin embargo, algunos trabajos también registran otros patrones diferentes al aquí reportado, por ejemplo Sun et al. [22] no encontraron una tendencia clara entre la elevación y la riqueza de especies de briófitos terrestres a lo largo de un gradiente altitudinal en zonas subtropicales alpinas del suroeste de China. Baniya et al. [23] documentaron una patrón en forma de campana de la riqueza de líquenes entre los 200-7400 m en diferentes formaciones vegetales, y Choudhary \& Singh [24] encontraron que la diversidad de especies de cianobacterias se correlacionó negativamente con la altitud en la región oriental de la India (Himalaya). Así, aunque nuestros resultados parecen ir en la línea de las especies criptógamas, no podemos aseverar que son resultados concluyentes y no se pueden hacer generalizaciones a nivel de ecosistema seco en Ecuador.

Nuestros resultados abren nuevas líneas de investigación (hipótesis 1 y 2) las cuales serán evaluadas en fu- 
turas investigaciones. Actualmente estamos adelantado un trabajo donde se incluyen variables climáticas y de suelos para ver cuáles son los factores que están influenciando la estructura y distribución las especies que conforman la CBS en el gradiente actitudinal seleccionado. Los resultados aquí documentados, aportan información adicional sobre el conocimiento de los patrones de diversidad y riqueza de especies que conforman la CBS en Ecuador. De esta forma, esperamos que nuestros resultados motiven a la comunidad científica, nacional e internacional a estudiar la CBS en Ecuador.

\section{Agradecimientos}

Los autores agradecen a J. Gusmán, M. Orellana, V. Rojas, F. Reyes-Bueno, D. Donoso y A. Cueva por su colaboración en alguna de las fases del proyecto. Esta investigación ha sido financiada por los proyectos PROY_CCNN_0021 y PROY_CCNN_852 con fondos de la Universidad Técnica Particular de Loja (UTPL).

\section{Referencias Bibliográficas}

[1] Belnap, J.; Lange, O., 2003. Biological Soil Crusts: Structure Function and Management. Springer-Verlag: Berlin.

[2] Soule, T.; Anderson, I.J.; Johnson, S.L.; Bates, S.T.; Garcia-Pichel, F. 2009. "Archaeal populations in biological soil crusts from arid lands in North America". Biología del Suelo y Bioquímica, 41(10):2069-2074. DOI: http://dx.doi. org/10.1016/j.soilbio.2009.07.023.

[3] Lange, O.L.; Kidron, G.; Büdel B.; Meyer, A.; Kilian, E.; Abeliovich, A.; 1992. "Taxonomic composition and photosynthetic characteristics of the "biological soil crusts covering sand dunes in the western Negev Desert". Functional Ecology, 6:519-527.

[4] Belnap, J. 2002. "Nitrogen fixation in biological soil crusts from southeast Utah, USA". Biology and Fertility of Soils, 35(2):128-135. DOI: http://dx.doi.org/10.1007/s00374-002-0452-x.

[5] Chaudhary, V.B.; Bowker, M.; O’Dell, T.E.; Grace, J.B.; Redman, A.E.; Rillig, M.C.; Johnson, N.C. 2009. "Untangling the biological contributions to soil stability in semiarid shrub- lands". Ecological Applications, 19(1):110-22. DOI: http://dx.doi.org10.1890/07-2076.1.

[6] Castillo-Monroy, A.P.; Maestre, F.T.; DelgadoBaquerizo, M.; Gallardo, A. 2010. "Biological soil crusts modulate nitrogen availability in semiarid ecosystems : insights from a Mediterranean grassland". Plant and Soil, 333(1-2):21-34. DOI: http://dx.doi.org/10.1007/s11104-009-0276-7.

[7] Castillo-Monroy, A.P.; Maestre, F.T.; Rey, A.; Soliveres, S.; García-Palacios, P. 2011. "Biological Soil Crust Microsites Are the Main Contributor to Soil Respiration in a Semiarid Ecosystem". Ecosystems, 14(5):835-847. DOI: http://dx.doi. org/10.1007/s10021-011-9449-3.

[8] Eldridge, D.; Bowker, M.; Maestre, F.; Alonso, P.; Mau, R.; Papadopoulos, J.; Escudero, A. 2010. "Interactive Effects of Three Ecosystem Engineers on Infiltration in a Semi-Arid Mediterranean Grassland". Ecosystems, 13(4):499-510. DOI: http://dx.doi.org/10.1007/s10021-0109335-4.

[9] Delgado-Baquerizo, M.; Covelo, F.; Maestre, F.T.; Gallardo, A. 2013. "Biological soil crusts affect small-scale spatial patterns of inorganic $\mathrm{N}$ in a semiarid Mediterranean grassland". Journal of Arid Environments, 91:147-150. DOI: http:// dx.doi.org/10.1016/j.jaridenv.2013.01.005.

[10] Prasse, R.; Bornkamm, R. 2000. "Effect of microbiotic soil surface crusts on emergence of vascular plants". Plant Ecology, 150:65-75. DOI: http://dx.doi.org/10.1023/A:1026593429455.

[11] DeFalco, L.A.; Detling, J.K.; Tracy, C.R.; Warren, S.D. 2001. "Physiological variation among native and exotic winter annual plants associated with microbiotic crusts in the Mojave Desert". Plant and Soil, 234(1):1-14. DOI: http://dx.doi. org/10.1023/A:1010323001006.

[12] Shepherd, U.; Brantley, S.; Tarleton, C. 2002. "Species richness and abundance patterns of microarthropods on cryptobiotic crusts in a piñonjuniper habitat: a call for greater knowledge". Journal of Arid Environments, 52:349-360. DOI: http://dx.doi.org/10.1006/jare.2002.1003. 
[13] Maestre, F.; Bowker, M.; Cantón, Y.; CastilloMonroy, A.P.; Cortina, J.; Escolar, C.; Escudero, A.; Lázaro, R.; Martínez, I. 2011. "Ecology and functional roles of biological soil crusts in semiarid ecosystems of Spain". Journal of Arid Environments, 75(12):1282-1291. DOI: http://dx.doi. org/10.1016/j.jaridenv.2010.12.008.

[14] Pointing, S.B.; Belnap, J. 2012. "Microbial colonization and controls in dryland systems". Nature Reviews Microbiology, 10(8):551-562. DOI: http://dx.doi.org/10.1038/nrmicro283.

[15] Rivera-Aguilar, V.; Montejano, G.; RodríguezZaragoza, S.; Durán-Díaz, A. 2006. "Distribution and composition of cyanobacteria, mosses and lichens of the biological soil crust of the Tehuacan Valley, Puebla, México". Journal of Arid Environments, 67:208-225. DOI: http://dx.doi. org/10.1016/j.jaridenv.2006.02.013.

[16] Büdel, B.; Darienko, T.; Deutschewitz, K.; Dojani, S.; Friedl, T.; Mohr, K.I.; Salisch, M.; Reisser, W.; Weber, B. 2009. "Southern African biological soil crusts are ubiquitous and highly diverse in drylands, being restricted by rainfall frequency". Microbial Ecology, 57(2):229-247. DOI: http://dx.doi.org/10.1007/s00248-0089449-9.

[17] Castillo-Monroy, A.P.; Maestre, F.T. 2011. "La costra biológica del suelo: Avances recientes en el conocimiento de su estructura y función ecológica". Revista chilena de historia natural, 84(1):1-21. DOI: http://dx.doi.org/10.4067/ S0716-078X2011000100001.

[18] Toledo, V.; Florentino de Andreu, A. 2012. "Evaluación de las propiedades biológicas y bioquímicas de la costra microbiotica de un suelo bajo vegetación natural en la región árida de Quíbor, Venezuela". Revista de investigación, 75: 143163.

[19] Lomolino, M.V. 2001. "Elevation gradients of species-density: historical and prospective views". Global Ecology and Biogeography, 10(1):3-13. DOI: http://dx.doi.org/10.1046/ j.1466-822x.2001.00229.x.
[20] Rahbek, C. 1995. "The elevational gradient of species richness: a uniform pattern?". Ecography, 18(2):200-205. DOI: http://dx.doi. org/10.1111/j.1600-0587.1995.tb00341.x.

[21] McCain, C.M.; Grytnes, J.A. 2010. "Elevation gradient in species richness". En: eLS. John Wiley \& Sons Ltd, Chichester. http://www.els.net., DOI: http://dx.doi.org/10.1002/9780470015902. a0022548.

[22] Sun, H.; Wu, Y.; Yu, D.; Zhou, J. 2013. "Altitudinal gradient of microbial biomass phosphorus and its relationship with microbial biomass carbon, nitrogen, and rhizosphere soil phosphorus on the eastern slope of Gongga Mountain, SW China". PLoS One, 8(9). e72952. DOI: http:// dx.doi.org/10.1371/journal.pone.0072952.

[23] Baniya, C.B.; Solhøy, T.; Gauslaa, Y.; Palmer, M.W. 2010. "The elevation gradient of lichen species richness in Nepal". Lichenologist, 42(1):83-96. DOI: http://dx.doi.org/10.1017/ S0024282909008627.

[24] Choudhary, K.K.; Singh, R.K. 2013. "Cyanobacterial diversity along altitudinal gradient in Eastern Himalayas of India”. Journal of alga biomass utilization, 4(2):53-58.

[25] Grime, J.P. 1973. "Control of species density in herbaceous vegetation". Journal of Environmental Management, 1:151-167.

[26] Espinosa, C.I.; Luzuriaga, A.L.; de la Cruz, M.; Montero, M.; Escudero, A. 2013. "Co-occurring grazing and climate stressors have different effects on the total seed bank when compared to the persistent seed bank". Journal of Vegetation Science, 24(6):1098-1107. DOI: http://dx.doi. org/10.1111/jvs.12043.

[27] Cabrera, O.; Prina, A. 2013. "Euphorbia weberbaueri (Euphorbiaceae), nuevo registro para Ecuador". Boletín de la Sociedad Argentina de Botánica, 48(1):137-141. 
[28] Sierra, R. 1999. "Propuesta preliminar de un sistema de clasificación de vegetación para el Ecuador Continental'. Rimana: Quito.

[29] Maestre, F.; Escudero, A.; Martinez, I.; Guerrero, C.; Rubio, A. 2005. "Does spatial pattern matter to ecosystem functioning?". Functional Ecology, 19:566-573. DOI: http://dx.doi. org/10.1111/j.1365.

[30] Martinez, I.; Escudero, A.; Maestre, F.; de la Cruz, A.; Guerrero, C.; Rubio, A. 2006. "Smallscale patterns of abundance of mosses and lichens forming biological soil crusts in two semiarid gypsum environments". Australian Journal of Botany, 54(4):339-348. DOI: http://dx.doi. org/10.1071/BT05078.

[31] Bowker, M.A.; Johnson, N.C.; Belnap, J.; Koch, G.W. 2008. "Short-term monitoring of aridland lichen cover and biomass using photography and fatty acids". Journal of Arid Environments, 72(6):869-878. DOI: http://dx.doi.org/10.1016/j. jaridenv.2007.11.006.

[32] Gradstein, S.R.; Churchill, S.P.; Salazar, A.N. 2001. "Guide to the Bryophytes of tropical America". Memoirs of the New York Botanical Garden, 86:1-577.

[33] León-Yánez, S.; Gradstein, S.R.; Wegner, C. 2006. "Hepáticas (Marchantiophyta) y Antoceros (Anthocerotophyta) del Ecuador, catálogo”. Publicaciones del Herbario QCA, Pontificia Universidad Católica del Ecuador, Quito.

[34] Churchill, S.P.; Griffin, D.; Muñoz J. 2000. A Checklist of the mosses of the tropical Andean countries. Ruizia, 17:1-203.

[35] Nash III, T.H.; Ryan, B.D.; Gries, C.; Bungartz, F. (eds.) 2002. "Lichen Flora of the Greater Sonoran Desert Region". Vol. I. Tempe: Lichens Unlimited, pp. 532.

[36] Colwell, R.K. 2013. "Statistical estimation of species richness and share species from samples". Enlace: http://viceroy.eeb.uconn.edu/estimates/
[37] Benítez, A.; Prieto, M.; González, Y.; Aragón, G. 2012. "Effects of tropical montane forest disturbance on epiphytic macrolichens". Science of the Total Environment, 441:169-175. DOI: http:// dx.doi.org/10.1016/j.scitotenv.2012.09.072.

[38] Concostrina-Zubiri, L.; Martínez, I.; Rabasa, S.; Escudero, A. 2014. "The influence of environmental factors on biological soil crust: from a community perspective to a species level approach". Journal of vegetation science, 25(2):503-513. DOI: http://dx.doi.org/10.1111/ jvs. 12084 .

[39] Belnap, J.; Phillips, S.L.; Troxler, T. 2006. "Soil lichen and moss cover and species richness can be highly dynamic: The effects of invasion by the annual exotic grass Bromus tectorum, precipitation, and temperature on biological soil crusts in SE Utah". Applied Soil Ecology, 32(1):63-76. DOI: http://dx.doi.org/10.1016/j. apsoil.2004.12.010.

[40] Gómez, D. A.; Aranibar, J.N.; Tabeni, S.; Villagra, P.E.; Garibotti, I.A.; Atencio, A. 2012. "Biological soil crust recovery after long-term grazing exclusion in the Monte Desert (Argentina). Changes in coverage, spatial distribution, and soil nitrogen". Acta Oecologica, 38:33-40. DOI: http://dx.doi.org/10.1016/j.actao.2011.09.001.

[41] Iturriaga, G.; Gaff, D.; Zentella, R. 2000. "New desiccation-tolerant plants, including a grass, in the central highlands of Mexico, accumulate trehalose". Australian Journal of Botany, 48(2):153-158. DOI: http//dx.doi.org/10.1071/ BT98062.

[42] Zhao, J.; Zheng, Y.; Zhang, B.; Chen, Y.; Zhang, Y. 2009. "Progress in the study of algae and mosses in biological soil crusts". Frontiers of Biology in China, 4(2):143-150. DOI: http:// dx.doi.org/10.1007/s11515-008-0104-0.

[43] Ullmann, I.; Büdel, B. 2003. "Ecological determinants of species composition of biological soil crusts on a landscape scale". Biological soil crusts: structure, function, and management. Springer-Verlag: Berlin. 
[44] Garcia-Pichel, F.; López-cortés, A.; Nubel, U. 2001. "Phylogenetic and morphological diversity of cyanobacteria in soil desert crusts from the Colorado Plateau phylogenetic and morphological diversity of cyanobacteria in soil desert crusts from the Colorado Plateau". Applied and Environmental Microbiology, 67(4):1902-1910. DOI: http//dx.doi.org/10.1128/AEM.67.4.1902.

[45] Austrheim, G. 2002. "Plant diversity patterns in semi-natural grasslands along an elevational gradient in southern Norway". Plant Ecology, 161(2): 193-205. DOI: http://dx.doi. org/10.1023/A:1020315718720.

[46] Bruun, H.H.; Moen, J.; Virtanen, R.; Grytnes, J.A.; Oksanen, L.; Angerbjörn, A. 2006. "Effects of altitude and topography on species richness of vascular plants, bryophytes and lichens in alpine communities". Journal of Vegetation Science, 17(1): 37-46. DOI: http://dx.doi. org/10.1111/j.1654-1103.2006.tb02421.x. 